\title{
RUTINAS EMOCIONALES EN FAMILIAS DE INFANTES VÍCTIMAS DE ACOSO: ESTUDIO DE CASO EN ESCUELA DE ZONA DE RIESGO
}

\author{
Rubén García Cruz \\ Universidad Autónoma del Estado de Hidalgo \\ rubengarciacruz2014@gmail.com \\ Citlali Hernández Sánchez \\ Universidad Autónoma del Estado de Hidalgo \\ citlahesa@gmail.com
}

Fecha de Recepción: 30 Junio 2019

Fecha de Admisión: 25 Septiembre 2019

\section{RESUMEN}

La presente investigación es de tipo mixta, basada en estudio de caso, la cual se dividió en dos fases, la primera de tipo exploratorio-descriptiva cuyo objetivo fue describir el fenómeno de acosovictimización a través de la Escala de ERAVE, compuesta por tres subescalas, en infantes escolarizados en zona de riesgo en la primaria Margarita Maza de Juárez, San Agustín Tlaxiaca, Hidalgo, misma que fue aplicada a 38 estudiantes. La segunda con enfoque etnográfico educativo, cuyo propósito fue analizar los discursos obtenidos mediante la entrevista semiestructurada en 3 escolares con puntajes altos en la escala de victimización, estos permitieron profundizar en la realidad familiar y sociocultural de los infantes y su comportamiento en la escuela. Los resultados indican que a mayor calidad de las rutinas emocionales en la familia, menor es la probabilidad que estén inmersos en el fenómeno acoso-victimización, manifestando niveles normales de ansiedad y angustia. Los sujetos de estudio están inmersos en una dinámica familiar violenta. Es así que las rutinas emocionales son poco sistematizadas y los vínculos superficiales en estas familias. Cuando se basan en emociones positivas, potencian factores de protección que favorecen la resiliencia y salud mental.

Palabras clave: rutinas emocionales; familia; acoso-victimización; funcionalidad familiar; escolares en zona de riesgo.

\section{ABSTRACT}

Emotional routes in families of victims of harassment: case study in school of risk area. The present investigation is of mixed type, based on case study, which was divided into two phases, the first exploratory-descriptive type whose objective was to describe the phenomenon of harassmentvictimization through the ERAVE Scale, composed of three subscales, in infants enrolled in a risk 
zone at "Margarita Maza de Juárez" Elementary, San Agustín Tlaxiaca, Hidalgo, which was applied to 38 students. The second with an ethnographic educational approach, whose purpose was to analyze the discourses obtained through the semi-structured interview in 3 schoolchildren with high scores on the victimization scale, these allowed to deepen the family and socio-cultural reality of the infants and their behavior in school. The results indicate that the higher the quality of emotional routines in the family, the lower the probability that they are immersed in the harassmentvictimization phenomenon, manifesting normal levels of anxiety and anguish. The study subjects are immersed in a violent family dynamic. Thus, emotional routines are little systematized and superficial ties in these families. When based on positive emotions, they enhance protective factors that favor resilience and mental health.

Keywords: emotional routines; family; harassment-victimization; family functionality; Schoolchildren in risk zone.

\section{INTRODUCCIÓN}

En investigaciones recientes realizadas en América Latina, se afirma desde datos epidemiológicos un incremento en la población escolarizada de bullying en sus diversas manifestaciones. Los principales hallazgos de dicha revisión fue la existencia de 51 estudios que indican una mayor prevalencia de este fenómeno sobre otros, con alta significancia en varones. Enfatizan sobre la necesidad de profundizar en el tema e implementar programas de prevención e intervención en las etapas de infancia, adolescencia y juventud (Garaigordobil, Mollo-Torrico, y Larrain, 2019). De acuerdo a la Organización para la Cooperación y el Desarrollo Económicos (OCDE), México ocupa el primer lugar internacional de casos de acoso escolar en educación básica, lo que afecta a 18 millones 781 mil 875 alumnos de primaria y secundaria de escuelas públicas y privadas (Milenio, 2018).

El acoso escolar es definido como una conducta dañina e intencional, realizada de forma repetida por una 0 más personas, y dirigida hacia una víctima a la que le resulta difícil defenderse (Olweus, 1993). Este fenómeno en ámbitos escolarizados tiene impacto negativo grave en las víctimas, quienes manifiestan mayores sentimientos de soledad, depresión y ansiedad, lo que repercute en baja autoestima (Cava, 2011).

Las investigaciones realizadas por Puértolas y Montiel (2017) reconocen que el perfil psicológico en menores implicados en situaciones de bullying está asociado al desequilibrio de poder, la indefensión de la víctima, la conducta hostil del agresor y la reiteración de la conducta. Estos aspectos se vinculan estrechamente con la debilidad física, psicológica y déficits sociales como son la impopularidad, escasez de relaciones sociales, incapacidad en la resolución de conflictos, condiciones que los pone en desventaja con los agresores. Por otro lado confirman estos estudios que la inseguridad, el miedo y la autopercepción negativa están asociados a carencias emocionales que provienen del contexto familiar y repercuten en los ámbitos escolarizados, desde problemas de rendimiento escolar hasta comportamientos suicidas y autolesivos, problemas de sueño, depresión, ansiedad y sentimiento de inseguridad, entre otros.

Los infantes víctimas de intimidación pertenecen a familias con cierta disfuncionalidad, por tanto no se deben analizar como un ente aislado, sino como parte de un conjunto en el que se ven inmersos factores tanto individuales como parentales y académicos (Cerezo, Ruiz Sánchez, y Arense, 2018). Múltiples son los factores familiares implicados en este fenómeno: conflictos intraparentales, violencia entre padres, rupturas parentales, maltrato, padres distantes y poco cálidos, castigos físicos e inconscientes, victimización entre hermanos, padres con bajo nivel escolar y proveniente de familias acosadoras (Arroyave, 2012).

Si tomamos como base el modelo ecológico, existen instancias que impactan de manera directa a la presencia de acoso escolar, entre ellas se encuentra la familia, la escuela y el grupo de igua- 
les. Las familias se convierten entonces en el primer espacio de crianza donde los cambios que generan tensión se encuentran directamente relacionados con problemas de comportamiento de los infantes en el ámbito escolar, por lo que un adecuado clima familiar inhibe el que los actos de violencia se presenten, puesto que la familia es el eje que conduce modelos de conducta y convivencia, modelos en los cuales los niños aprenden el grado de tolerancia a la agresión que es adecuado (Ovejero, 2014).

Esta funcionalidad debe de ser vista como un estado variable y modificable en el tiempo, donde no solo se incluye a la infancia temprana, dicho de otro modo es un estado emocionalmente equilibrado de la familia. Sin embargo, en el caso de las familias funcionales se ha demostrado que son capaces de crear un entorno de bienestar emocional que facilita el desarrollo de sus miembros, con un bajo grado de discrepancia y responsable a las necesidades de cada integrante. Por tanto no sufren crisis, ni trastornos psicológicos graves, es por ello que la funcionalidad se asocia al sistema familiar en cuanto a los niveles de cohesión, flexibilidad, comunicación, estrés, conflictos emociones y vínculos (Fuentes y Merino, 2016).

Las rutinas emocionales como vínculos positivos generados dentro de la familia, permiten que exista funcionalidad, favorece al crecimiento tanto individual, familiar y social, puesto que se considera como un sistema abierto compuesto por subsistemas, vistos estos como los integrantes, quienes se encargaran de desempeñar funciones, roles y tareas claras que les permite un ajuste al medio extra familiar. Las rutinas y sus rituales en la familia han sido tema de interés para comprender la conducta de los infantes escolarizados, sobre todo en esta época en que existe una gran diversidad de tipos de familias.

Perdura una gran preocupación para entender la asociación entre rutinas y rituales adecuados que provienen de historias y tradiciones familiares, así como crear ideas innovadoras para atender las problemáticas emocionales de los infantes. Se ha comprobado que habituar a los niños y niñas en ciertas rutinas, sobre todo las de tipo emocional, favorecen al desarrollo individual y al crecimiento personal. Los estudiosos de las rutinas emocionales en la familia consideran que profundizar en las relaciones diádicas puede contribuir a entender el comportamiento de los infantes en otros contextos como la escuela; por otra parte, consideran que indagar acerca de las relaciones maritales, aportará aún más conocimientos sobre el comportamiento de los hijos en ámbitos escolares. La formas en que se solucionan los conflictos de pareja en el ámbito del hogar influyen en como los niños regulan su propio afecto y bajo ciertas condiciones los problemas de conductas (Fiese, 2006).

En estudios realizados por Muñiz, Silver y Stein (2014) que tenían como propósito la intervención en niños de primera infancia con rutinas familiares y su efecto sobre la salud socio-emocional, encontraron que por cada rutina familiar adicional en la que el niño participa hay mayor probabilidad de presentar mayor salud socio-emocional. Las rutinas empleadas fueron: canto, juego, cuento de historias. Afirman que promover las rutinas familiares (dentro de ellas las emocionales), que van desde los saludos, abrazos, besos y juegos sistematizados y estructurados habitualmente, contribuyen a un incremento del bienestar antes de ingresar a la vida escolar.

\section{OBJETIVOS DE LA INVESTIGACIÓN}

La presente investigación fue dividida en dos fases, la primera fase tuvo como objetivo fue describir el fenómeno de acoso-victimización a través del ERAVE, en infantes escolarizados en zona de riesgo en la primaria "Margarita Maza de Juárez" de la comunidad "El Durazno", de San Agustín Tlaxiaca, Hidalgo, México. El objetivo de la segunda fase fue analizar los discursos obtenidos mediante la entrevista semiestructurada en escolares de puntajes altos en victimización (a través del ERAVE) con el propósito de analizar la expresión y sistematicidad de las rutinas emocionales. 


\section{MUESTRA Y/O PARTICIPANTES}

La muestra estuvo conformada por 38 alumnos de $5^{\circ}$ y $6^{\circ}$ grado, a los cuales se les aplicó de forma grupal la Escala de Reynolds de Acoso Victimización para Escolares (ERAVE). Para la segunda fase, fueron seleccionados tres de los 38 estudiantes cuyos puntajes obtenidos durante la aplicación fueron altos en la escala de victimización.

\section{METODOLOGÍA Y/O INSTRUMENTOS UTILIZADOS}

La investigación fue realizada desde una metodología mixta, basada en un estudio de caso con enfoque de etnografía educativa. Se dividió en dos fases, la primera de tipo exploratoria-descriptiva, durante la cual se aplico de manera grupal a los 38 alumno de 5to y 6to, la Escala de Reynolds de Acoso Victimización para Escolares (ERAVE).

Este instrumento se encuentra dividido en tres subescalas: 1. Escala de Acoso- Victimización (EAV), cuyo objetivo es medir el comportamiento de intimidación y acoso entre iguales en o cerca de las escuelas, 2. Escala de Angustia Acoso-Victimización (EAAV) que evalúa dimensiones de la angustia psicológica especifica de los estudiantes acosados, 3. Escala de Ansiedad por Violencia Escolar (EAVE) evalúa la ansiedad del estudiante respecto de las escuelas como entornos peligrosos 0 amenazantes. La escala completa se califica con base a una puntuación que va del nunca (cero puntos) hasta cinco o más veces (tres puntos) y en los puntajes de gravedad clínica va desde normal hasta grave.

Lo anterior, se llevó a cabo posterior al planteamiento de la problemática educativa propuesta desde una institución de gobierno, posteriormente se realizó un acercamiento a la escuela para determinar las necesidades escolares, organizar prioridades: reunión con directivos, docentes y padres, siguiendo un protocolo ético.

La segunda fase fue analizar los discursos obtenidos mediante la entrevista semiestructurada en escolares de puntajes altos en victimización (a través del ERAVE) con el propósito de analizar desde la perspectiva cualitativa con enfoque etnográfico la expresión y sistematicidad de las rutinas emocionales en la familia de los tres casos seleccionados, las mismas fueron grabadas en audio y video para luego ser transcritas y analizadas. El último paso de la segunda fase pretende interpretar expresiones no verbales bajo el fotograma de Eckmann.

La metodología descrita anteriormente se detalla en la Figura 1. 
Figura 1. Esquema general de investigación.

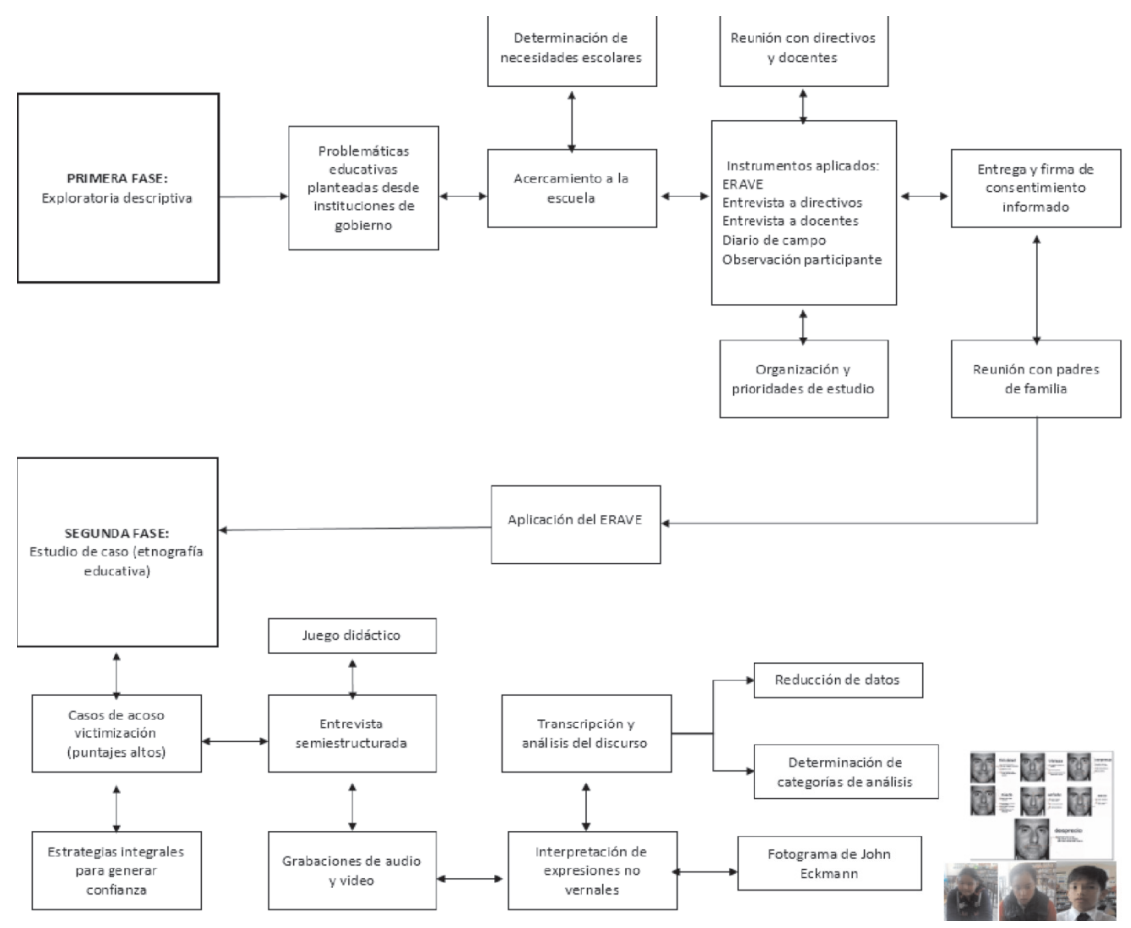

\section{RESULTADOS ALCANZADOS}

\section{Fase uno}

En la Escala de Acoso, el $84.21 \%$ de los alumnos presentaron niveles normales de acoso, el $7.84 \%$ niveles clínicamente significativos, el $2.63 \%$ moderadamente grave y el $5.26 \%$ clínicamente grave.

Tabla 1. Escala de Acoso

\begin{tabular}{ccc|}
\hline Puntajes de gravedad clínica & No. de infantes & Porcentaje \\
\hline Normal & 32 & 84.21 \\
\hline Clínicamente significativo & 3 & 7.89 \\
\hline Moderadamente grave & 1 & 2.63 \\
\hline Grave & 2 & 5.26 \\
\hline Total & 38 & \\
\hline
\end{tabular}

En la Escala de Victimización el $73.68 \%$ de los alumnos presentaron niveles normales de victimización, el 5.26\% niveles clínicamente significativos, el $7.89 \%$ modernamente grave y el $13.16 \%$ grave. 
RUTINAS EMOCIONALES EN FAMILIAS DE INFANTES VÍCTIMAS DE ACOSO:

ESTUDIO DE CASO EN ESCUELA DE ZONA DE RIESGO

Tabla 2. Escala de Victimización

\begin{tabular}{lrr|}
\hline Puntajes de gravedad clínica & No. De infantes & \multicolumn{2}{c|}{ Porcentaje } \\
\hline Normal & 28 & 73.68 \\
\hline Clínicamente significativo & 2 & 5.26 \\
\hline Moderadamente grave & 3 & 7.89 \\
\hline Grave & 5 & 13.16 \\
\hline Total & 38 & \\
\hline
\end{tabular}

En la Escala de Angustia Internalizada, el $76.32 \%$ presenta niveles normales, el 7,89\% niveles clínicamente significativos, moderadamente grave y grave.

Tabla 3. Escala de Angustia Internalizada

\begin{tabular}{lrrr|}
\hline & & \\
\hline Puntajes de gravedad clínica & No. De infantes & \multicolumn{2}{c|}{ Porcentaje } \\
\hline Normal & 29 & 76.32 \\
\hline Clínicamente significativo & 3 & 7.89 \\
\hline Moderadamente grave & 3 & 7.89 \\
\hline Grave & 3 & 7.89 \\
\hline Total & 38 & \\
\hline
\end{tabular}

En la Escala de Angustia Externalizada, el $78.95 \%$ de los alumnos presentaron niveles normales, el $13.16 \%$ niveles clínicamente significativo, el $2.63 \%$ moderadamente grave y el $5.26 \%$ clínicamente graves.

Tabla 4. Escala de Angustia Externalizada

\begin{tabular}{lcc}
\hline Puntajes de gravedad clínica & No. de infantes & Porcentaje \\
\hline Normal & 30 & 78.95 \\
\hline Clínicamente significativo & 5 & 13.16 \\
\hline Moderadamente grave & 1 & 2.63 \\
\hline Grave & 2 & 5.26 \\
\hline Total & 38 & \\
\hline
\end{tabular}

Para la Escala de Angustia Total, el $63.16 \%$ de los estudiantes presentaron niveles normales, el $18.42 \%$ clínicamente significativo, el $7.89 \%$ moderadamente grave y el $10.53 \%$ clínicamente grave.

Tabla 5. Escala Angustia Total

\begin{tabular}{lrr}
\hline Puntajes de gravedad clínica & No. De infantes & Porcentaje \\
\hline Normal & 24 & 63.16 \\
\hline Clínicamente significativa & 7 & 18.42 \\
\hline Moderadamente grave & 3 & 7.89 \\
\hline Grave & 4 & 10.53 \\
\hline Total & 38 & \\
\hline
\end{tabular}


En la Escala de Ansiedad por Violencia Escolar, el 65.79\% presentaron niveles normales, el 5.26 clínicamente significativo, el $2.63 \%$ moderadamente grave y el $26.32 \%$ clínicamente grave.

Tabla 6. Escala Ansiedad por Violencia Escolar

\begin{tabular}{lrr}
\hline Puntajes de gravedad clínica & No. De infantes & Porcentaje \\
\hline Normal & 25 & 65.79 \\
\hline Clínicamente significativo & 2 & 5.26 \\
\hline Moderadamente grave & 1 & 2.63 \\
\hline Grave & 10 & 26.32 \\
\hline Total & 38 & \\
\hline
\end{tabular}

\section{Fase dos}

En la entrevista semiestructurada se obtuvieron las siguientes categorías: reconocimiento de emociones, ausencia de los padres, disfuncionalidad familiar, violencia en la familia nuclear y extensa, sueños y deseos afectivos, roles, baja calidad en las rutinas emocionales (en la familia y en la escuela).

Los tres infantes representados como A, B y C tienen en común ser víctimas de acoso escolar, al igual que la categoría de violencia en la familia extensa. Los infantes A y B tienen en común la categoría de disfunción familiar y violencia en la familia nuclear dando como resultado en el ERAVE angustia total y ansiedad clínicamente grave. El infante $C$ presenta niveles de angustia total y ansiedad clínicamente normales.

Para una mayor compresión se explica con los siguientes esquemas:
$\mathbf{A}$
B
Víctimas de acoso escolar
$\left.\begin{array}{l}\text { A } \\ \text { B }\end{array}\right]$. Disfunción familiar, violencia en familia
C Violencia en familia extensa
B Angustia total y ansiedad grave
C: Angustia total y ansiedad en niveles normales

\section{DISCUSIÓN}

Los casos estudiados confirman que el abandono afectivo de familias disfuncionales repercute en la regulación emocional y por ende en la salud mental positiva y el bienestar. Esto se constata en el discurso siguiente:

...en algunas partes me siento triste porque no está aquí (...), él es el que debería estar aquí, no mi padrastro, él es el que me debería ver crecer y festejarme mis cumpleaños (...), la última vez si lo vi y pues él ya tiene una familia con otra señora y tiene dos hijas (A.P, 9 años, $4^{\circ}$ grado).

Las características económicas y socioculturales de la familia inducen al incremento de la violencia familiar y escolar: 
Pues él desarma piezas así como el cobre, y el aluminio y todo eso y lo lleva a vender, y así le llegan muchas cosas (...), trabaja igual que mi papá, los dos se van a trabajar (...), en algunos días no llegan y en otro si, llegan a las 20 a las 100 a las 11 de la noche (...). Me siento triste, porque pues no hay nadie, y me gustaría que estuviera mi mamá y mi hermano (A.P, 9 años, $4^{\circ}$ grado).

Asimismo, se presenta disfuncionalidad familiar la cual genera ciertas conductas antisociales:

...me llevo mal con mi papá, es que a mi prima le dice bonita y a mí me dice cosas malas "Ay, ahí viene la mugrosa" (...) mi prima es como su hija y nada más le compra lo que sea a ella y a mí no (D.H, 10 años, $4^{\circ}$ grado).

La discontinuidad educativa (afectiva y cognitiva) entre la escuela y familia incrementa el riesgo de acoso y victimización:

Nosotros nos enteramos por los niños de muchas cosas pero si los papás no quieren no podemos hacer nada (B.M, maestra de 2do grado)

\section{CONCLUSIONES}

Las rutinas emocionales favorecen a la funcionalidad familiar y, por otra parte, disminuyen el riesgo de estar inmerso en el fenómeno de acoso-victimización en espacios escolares. Asimismo, a mayor calidad de vínculos y rutinas emocionales sistematizadas en la familia es menor el nivel de angustia total y ansiedad, y estas influyen en el nivel de resiliencia y en la salud mental positiva. Es importante destacar que las características económicas y socioculturales de la familia inducen al incremento de la violencia familiar y escolar.

\section{REFERENCIAS BIBLIOGRÁFICAS}

Angeles, E. (18 de noviembre de 2014). "Tulancingo y Pachuca con los índices más altos de bullying". Quadratín Hidalgo. Disponible en: https://hidalgo.quadratin.com.mx/municipios/regiones/Tulancingo-y-Pachuca-con-los-indices-mas-altos-de-bullying/

Arroyave , P. (2012). "Factores de vulnerabilidad y riesgo asociados al bullying". CES Psicología, $5(1), 118-125$.

Bisquerra, R. (2004). Metodología de la investigación educativa. Editorial: La Muralla, S. A.

Jesús Cava, M. (2011). "Familia, profesorado e iguales: claves para el apoyo a las víctimas de acoso escolar". Psychosocial Intervention, 20(2), 183-192.

Puèrtolas, A. e I. Montiel (2017). "Bullying en la educación secundaria: una revisión sobre las características de las víctimas y las víctimas-acosadores y las consecuencias de su victimización". Revista de Victimología/Journal of Victimology, (5), 85-128.

Cerezo, F., C. Ruiz, C Sánchez y J. Arense (2018). "Dimensions of parenting styles, social climate, and bullying victims in primary and secondary education". Psicothema, 30 (1), p. 59 - 65. doi: 10.7334/psicothema2016.360

Dolores, M. (3 de agosto de 2017). Reporta SEPH 210 quejas por bullying. Síntesis. Recuperado de: https://hidalgo.sintesis.mx/2017/08/03/bulliyng-quejas-educacion-basica-hidalgo-seph/

Fiese, B. H. (2006). Family routines and rituals. Yale: University Press.

Fuentes, A. y J. Merino (2016). "Validación de un instrumento de Funcionalidad Familiar". Ajayu. Órgano de Difusión Científica del Departamento de Psicología de la Universidad Católica Boliviana "San Pablo", 14(12), 247-183.

Gallego, A. (2012). "Recuperación crítica de los conceptos de familia, dinámica familiar y sus características". Revista Virtual Universidad Católica del Norte, (35), 326 - 345.

Garaigordobil, M., J. P. Mollo-Torrico y E. Larrain (2019). "Prevalencia de bullying y cyberbullying en Latinoamérica: una revisión”. Revista Iberoamericana de Psicología, 11(3), 1-18. 
Hernández, R. (2001). Metodología de la investigación. México, D.F.: McGraw-Hill.

Maturana, G. y C. Garzón (2015). "La etnografía en el ámbito educativo: una alternativa metodológica de investigación al servicio docente". Revista de Educación y Desarrollo Social, 9(2), 192205.

Muñiz, E. I., E. J. Silver y R. E. Stein (2014). "Family routines and social-emotional school readiness among preschool-age children". Journal of Developmental \& Behavioral Pediatrics, 35(2), 9399.

Olweus, D. (1993). Bullying at school: What we know and what we can do. Oxford: Blackwell (traducción, 1998, Conductas de acoso y amenaza entre escolares. Madrid: Morata).

Ovejero, A. (2014). "Actores psicosociales y acoso escolar en el ámbito familiar". International Journal of Developmental and Educational Psychology, 5(1), 351-353

Quintero, Á. (2007). Diccionario especializado en familia y género. Buenos Aires: Lumen Humanitas. Milenio, Redacción (14 de agosto de 2018). “Bullying', el terror escolar en México”. Milenio. Recuperado de: https://www.milenio.com/ciencia-y-salud/bullying-el-terror-escolar-en-mexico

Reynolds, W. (2017). Escala Reynolds de Acoso Victimización para escolares, Ciudad de México, México: Manual Moderno

Secretaría de Educación Pública (2010). Informe nacional sobre género y violencia en la educación. México: SEP, UNICEF 
